\title{
Caracterização de propriedades tecnológicas de três folhosas deterioradas por térmitas
}

\author{
Three hardwoods technological properties \\ characterization deteriorated by termites
}

\author{
Ezequiel Gallio ${ }^{1}$, Paula Zanatta ${ }^{1}$, Sabrina Finatto Machado ${ }^{1}$, \\ Rafael Beltrame ${ }^{1}$, Darci Alberto Gatto ${ }^{1}$
}

\footnotetext{
${ }^{1}$ Programa de Pós-Graduação em Ciência e Engenharia de Materiais - Universidade Federal de Pelotas, Pelotas, Rio Grande do Sul, Brasil.

e-mail: egeng.florestal@gmail.com; zanatta_paula@hotmail.com; sabrinamfinatto@gmail.com; beltrame.rafael@yahoo.com.br; darcigatto@yahoo.com
}

\section{RESUMO}

O objetivo deste estudo foi determinar as propriedades tecnológicas das espécies florestais Corymbia maculata, Eucalyptus dunnii e Eucalyptus saligna, e verificar as modificações nas características destas madeiras após ensaio de preferência alimentar com térmitas Nasutitermes. O ensaio de preferência alimentar foi conduzido através da norma ASTM D 3345 (2008) adaptada, avaliando a perda de massa e as propriedades mecânicas no fim do período correspondente. Para obtenção dos resultados, fez-se análise da massa específica básica $\left(\mu_{\mathrm{EB}}\right)$, com o auxílio da norma ASTM D 143 (2014), dureza Janka nos planos tangencial e radial e o módulo de elasticidade $\left(\mathrm{E}_{\mathrm{C}}\right)$ e resistência à compressão $\left(\mathrm{F}_{\mathrm{C}}\right)$, obtidos pelo ensaio de compressão paralela às fibras, antes e após o ataque de térmitas. Acerca das propriedades tecnológicas, verificou-se que a espécie Corymbia maculata apresentou a maior $\mu_{\mathrm{EB}}\left(0,674 \mathrm{~g} / \mathrm{cm}^{3}\right)$ e resistência mecânica, com os maiores valores de dureza Janka em ambos os planos anatômicos (tangencial $=664,6 \mathrm{kgf} / \mathrm{cm}^{2}$; radial $=593,8 \mathrm{kgf} / \mathrm{cm}^{2}$ ) e parâmetros relacionados à compressão paralela às fibras $\left(\mathrm{E}_{\mathrm{C}}=294.873 \mathrm{kgf} / \mathrm{cm}^{2} ; \mathrm{F}_{\mathrm{C}}=667,7 \mathrm{kgf} / \mathrm{cm}^{2}\right)$. Após o ensaio com térmitas Nasutitermes, a maior perda de massa foi observada na espécie Eucalyptus dunnii, indicando assim a sua preferência alimentar. Estes ocasionaram decréscimos não significativos nas propriedades mecânicas, quando comparada às mensuradas antes do ensaio de preferência alimentar. A partir dos resultados obtidos, conclui-se que a deterioração das madeiras por Nasutitermes, em conjunto com as características anatômicas, ocasionaram variações nas propriedades tecnológicas analisadas, contudo, as espécies Eucalyptus saligna e Corymbia maculata mostraram resistência natural elevada em relação aos cupins.

Palavras-chave: preferência alimentar; Nasutitermes; deterioração da madeira; propriedades mecânicas.

\section{ABSTRACT}

The objective of this study was to determine technological properties of Corymbia maculata, Eucalyptus dunnii and Eucalyptus saligna forest species, and to verify the changes in the characteristics of these Woods after food preference assay with Nasutitermes termites. The food preference assay was conducted through of the standard ASTM D 3345 (2008) adapted, evaluating the mass loss and mechanical properties at the end of the corresponding period. To obtain the results, the basic specific mass $\left(\mu_{\mathrm{EB}}\right)$ was analyzed, with the aid of standard ASTM D 143 (2014), Janka hardness in the tangential and radial planes and the elasticity modulus $\left(\mathrm{E}_{\mathrm{C}}\right)$ and compression resistence $\left(\mathrm{F}_{\mathrm{C}}\right)$, both obtained by the compression parallel to grain test, before and after the termites attack. About the technological properties, it was verified that the Corymbia maculata species had the highest $\mu_{\mathrm{EB}}\left(0.674 \mathrm{~g} / \mathrm{cm}^{3}\right)$ and mechanical resistance, with the highest values of Janka hardness in both anatomical planes (tangential $=664.6 \mathrm{kgf} / \mathrm{cm}^{2}$, radial $=593.8 \mathrm{kgf} / \mathrm{cm}^{2}$ ) and parameters related to compression parallel to grain $\left(\mathrm{E}_{\mathrm{C}}=294.873 \mathrm{kgf} / \mathrm{cm}^{2}, \mathrm{~F}_{\mathrm{C}}=667.7 \mathrm{kgf} / \mathrm{cm}^{2}\right)$. After the Nasutitermes termites test, the greatest mass loss was observed in the Eucalyptus dunnii species, thus indicating its food preference. These caused insignificant decreases in the mechanical properties, when compared to those measured before the food preference assay. From the results obtained, it is concluded that the wood deterioration by $\mathrm{Na}$ - 
sutitermes, together with the anatomical characteristics, caused variations in the technological properties analyzed, however, the species Eucalyptus saligna and Corymbia maculata showed high natural resistance in relation to the termites.

Keywords: food preference; Nasutitermes; wood deterioration; mechanical properties.

\section{INTRODUÇÃO}

A madeira é considerada um material orgânico suscetível a deterioração, que pode ser causada por agentes bióticos e abióticos, e dependendo das condições, pode sofrer diferentes níveis de danos. A consequente redução dos parâmetros relacionados à qualidade desse material em função da alteração das propriedades intrínsecas provocada por esses agentes ocasionam grandes prejuízos sociais e econômicos, que levam a substituição e reposição dessas peças.

Quando a madeira está em contato com o solo ou em diferentes teores de umidade, os cupins ou térmitas recebem destaque significativo, pois apresentam facilidade de desenvolvimento quando em condições favoráveis [1] e causam consideráveis danos a madeira. Em especial, os cupins do gênero Nasutitermes apresenta grande diversidade de espécies, com ampla distribuição geográfica e atacam diferentes classes de madeira [2].

A resistência natural da madeira em relação a deterioração causada por organismos xilófagos varia entre as espécies florestais e isto depende das propriedades que estas apresentam [3]. Assim, por meio de estudos prévios que utilizam ensaios de preferência alimentar destes insetos, é possível verificar a suscetibilidade que a madeira apresenta à biodeterioração e as possíveis alterações do ataque causado às propriedades tecnológicas desta, as quais possuem relação direta com a qualidade do produto. Com isso, há embasamento científico para selecionar espécies de maior durabilidade e que se adaptam a condições determinadas, acarretando em redução de custos com tratamentos preservantes.

Dentre as madeiras comercializadas, os gêneros Corymbia e Eucalyptus recebem uma atenção especial do setor madeireiro nacional em função do fornecimento de matéria-prima para a fabricação de produtos [4]. Isto se deve a elevada produtividade em curto espaço de tempo e em diferentes condições climáticas, além da qualidade e boa forma [5].

A empregabilidade dessas madeiras é destinada para diversas finalidades, tais como, produção de celulose e papel, carvão vegetal, lenha e até mesmo madeira sólida [6]. Contudo, visando a utilização racional e adequada dessas espécies, faz-se necessário o conhecimento das suas propriedades tecnológicas [7]. LOGSDON et al. [8] descrevem que a utilização da madeira para fins estruturais necessita um prévio conhecimento das propriedades mecânicas para a recomendação da espécie adequada.

Através do exposto acima, o objetivo deste estudo foi efetuar a caracterização de propriedades tecnológicas das espécies Corymbia maculata, Eucalyptus dunnii e Eucalyptus saligna, pertencentes à família Myrtaceae, e analisar a influência da deterioração causada pelo ataque de térmitas do gênero Nasutitermes.

\section{MATERIAIS E MÉTODOS}

\subsection{Material utilizado}

As madeiras utilizadas para o estudo foram Corymbia maculata, Eucalyptus dunnii, Eucalyptus saligna, 22, 28 e 22 anos de idade, respectivamente, e a espécie Pinus elliottii (com aproximadamente 15 anos de idade), que serviu de referência no ensaio de preferência alimentar com térmitas aplicado.

Para tanto, a partir das tábuas das referidas espécies, confeccionaram-se corpos de prova com dimensões de 2,0 x 2,0 x 15,0cm (tangencial x radial x longitudinal). Após dimensionados, os mesmos ficaram acondicionados em sala climatizada controlada $\left(20^{\circ} \mathrm{C}\right.$ de temperatura e $65 \%$ de umidade relativa do ar) até que atingissem equilíbrio higroscópico de $12 \%$ de umidade. Após estabilizados, os parâmetros de massa e volume iniciais foram adquiridos, por meio do uso de uma balança de precisão de $\pm 0,01 \mathrm{~g}$ e paquímetro digital.

\subsection{Ensaio de preferência alimentar}

No ensaio de preferência alimentar, empregaram-se térmitas do gênero Nasutitermes sp. O ensaio de biodeterioração foi conduzido conforme adaptação da norma ASTM D 3345 [9], em uma caixa da água de capacidade volumétrica de 2.000 litros, preenchida com uma camada de $15 \mathrm{~cm}$ de areia, a qual foi periodicamente umedecida com água, permitindo um ambiente favorável ao desenvolvimento dos cupins, como o demons- 
trado na Figura 1.

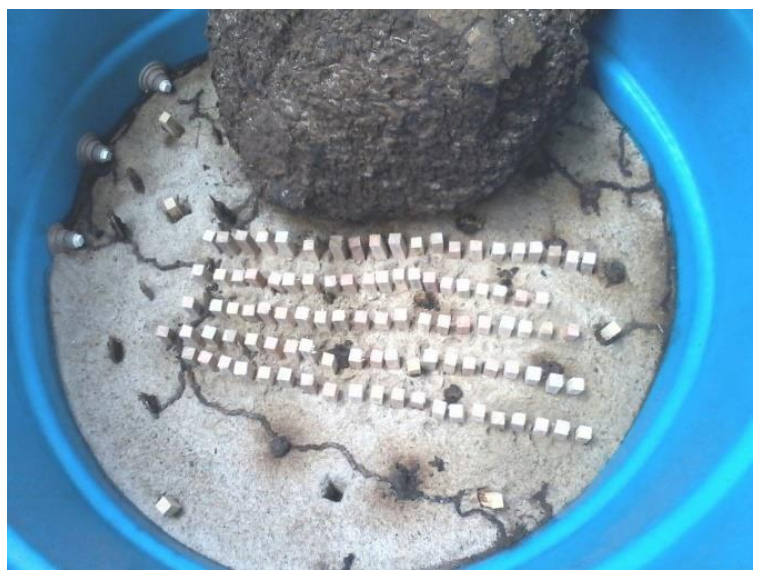

Figura 1: Experimento com térmitas Nasutitermes e disposição dos corpos de prova.

Os corpos de prova das diferentes espécies citadas acima, ficaram expostos à deterioração por um período de 40 dias. Na sequência do experimento, utilizando um pincel, removeu-se de maneira cuidadosa o substrato (areia) e os cupins remanescentes nos corpos de prova. Em seguida, encaminhou-se os mesmos até a sala climatizada controlada que atingissem umidade de equilíbrio de $12 \%$, o que possibilitou a determinação da perda de massa e avaliação das alterações das demais propriedades tecnológicas de interesse.

\subsection{Propriedades tecnológicas antes e após o ataque de térmitas}

Antes do ensaio de biodeterioração, determinou-se a massa específica básica $\left(\mu_{\mathrm{EB}}\right)$ das madeiras estudadas. Para tanto, determinou-se as dimensões dos corpos de prova em estado saturado de umidade, as quais possibilitaram obter as dimensões e volume saturado, pelo método estereométrico.

Sequencialmente, com o auxílio de uma estufa laboratorial (ajustada à $103 \pm 2{ }^{\circ} \mathrm{C}$ de temperatura), secaram-se as amostras até a estabilização da massa. Essas etapas e o emprego da Equação 1 abaixo possibilitaram a determinação da propriedade física de interesse.

$\mu_{E B}=M_{0} / V_{S}$

Em que: $\mu_{E B}=$ massa específica básica $\left(\mathrm{g} / \mathrm{cm}^{3}\right) ; M_{0}=$ massa da amostra seca em estufa à $103 \pm 2^{\circ} \mathrm{C}(\mathrm{g})$; $V_{S}=$ volume da amostra em condição saturada de umidade $\left(\mathrm{cm}^{3}\right)$.

Em referência as propriedades mecânicas, os parâmetros de resistência à compressão $\left(\mathrm{F}_{\mathrm{C}}\right)$ e a módulo de elasticidade $\left(\mathrm{E}_{\mathrm{C}}\right)$ pelo ensaio de compressão paralela às fibras, e dureza Janka nos planos radial e tangencial, foram determinados com base na adaptação da norma ASTM D 143 [10] antes e após o ataque das térmitas. Os parâmetros de interesse foram obtidos em uma máquina universal de ensaios (EMIC), equipada com célula de carga de $300 \mathrm{kN}$ e sistema de aquisição de dados computadorizado.

Por fim, avaliou-se a influência da deterioração causada pelo ataque de térmitas do gênero Nasutitermes nas madeiras de Corymbia maculata, Eucalyptus dunnii, Eucalyptus saligna por meio da determinação da perda de massa (PM), conforme a Equação 2.

$$
P M=((M 1-M 2) / M 1) * 100
$$

Em que: $\mathrm{PM}=$ perda de massa da madeira após o ataque de térmitas (\%); $\mathrm{M}_{1}=$ massa da amostra antes do ensaio $(\mathrm{g}) ; \mathrm{M}_{2}=$ massa da amostra após o ensaio $(\mathrm{g})$;

\subsection{Análises estatísticas dos dados}

Visando a obtenção da homogeneidade das variâncias dos dados, transformaram-se os valores percentuais de perda de massa em Arcsen $\sqrt{\mathrm{PM} / 100}$, conforme sugestão de STEELL e TORRIE [11]. Após, utilizando-se o software Statgraphics, submeteram-se os valores médios de parâmetros tecnológicos de interesse à análise da variância e posterior teste de comparação de médias pelo teste LSD Fisher, com 5\% de probabilidade de erro. 


\section{RESULTADOS E DISCUSSÃO}

\subsection{Propriedades físicas e mecânicas das madeiras}

$\mathrm{Na}$ Tabela 1 verifica-se a existência de diferença significativa entre as massas específicas básicas $\left(\mu_{\mathrm{EB}}\right)$ das três espécies florestais. O maior valor de massa específica foi encontrado para a madeira de Corymbia maculata, contudo, inferior ao encontrado por MARTINS et al. [12], com idade superior a 30 anos $\left(0,805 \mathrm{~g} / \mathrm{cm}^{3}\right)$.

Para as madeiras de Eucalyptus dunnii e Eucalyptus saligna, o valor de $\mu_{\mathrm{EB}}$ é semelhante e superior ao verificado por BATISTA et al. [13] em seus estudos com clones de 11 anos de idade para as referidas espécies $\left(0,56 \mathrm{~g} / \mathrm{cm}^{3}\right.$ e $0,46 \mathrm{~g} / \mathrm{cm}^{3}$, respectivamente). Considerando a classificação proposta por CARVALHO [14], a Corymbia maculata enquadra-se como uma madeira moderadamente pesada $\left(0,65-0,79 \mathrm{~g} / \mathrm{cm}^{3}\right)$, enquanto as espécies de Eucalyptus dunnii e Eucalyptus saligna, ambas inserem-se na classe de madeiras leves $(0,50$ $\left.0,64 \mathrm{~g} / \mathrm{cm}^{2}\right)$.

Tabela 1: Resumo estatístico e valores médios para a massa específica básica das espécies florestais estudadas.

\begin{tabular}{cc}
\hline Espécie & $\boldsymbol{\mu}_{\mathrm{EB}}\left(\mathbf{g} / \mathbf{c m}^{3}\right)$ \\
\hline Corymbia maculata & $0,674(0,021) \mathrm{a}$ \\
\hline Eucalyptus dunnii & $0,573(0,048) \mathrm{c}$ \\
\hline Eucalyptus saligna & $0,632(0,019) \mathrm{b}$ \\
\hline $\mathrm{CV}(\%)$ & 8,21 \\
\hline $\mathrm{F}$ & $24,58^{*}$
\end{tabular}

Em que: valores entre parênteses apresentam o desvio padrão $\left(\mathrm{g} / \mathrm{cm}^{3}\right)$. Médias nas colunas acompanhadas de mesma letra não diferem entre si, conforme teste LSD Fisher, em 5\% de probabilidade de erro. $*$ = difere estatisticamente $(\mathrm{P}<0,05)$; ns $=$ não difere estatisticamente $(\mathrm{P}>0,05)$;

Além da idade, parâmetros anatômicos como tipo de lenho (inicial ou tardio), posição no fuste, porosidade e dimensões das fibras podem influenciar na massa específica da madeira, no que tange a mesma ou diferentes espécies florestais. TREVISAN et al. [15] complementam que essa propriedade física $\left(\mu_{\mathrm{EB}}\right)$ correlaciona-se diretamente com a maioria das propriedades mecânicas da madeira, enquanto MATTOS et al. [16] ressaltaram a importância do conhecimento acerca da $\mu_{\mathrm{EB}}$, pois a mesma está diretamente atrelada com a qualidade da madeira.

Logo, na Tabela 2, verifica-se a ausência de diferenças significativas para os parâmetros de resistência à compressão $\left(\mathrm{F}_{\mathrm{C}}\right)$ e módulo de elasticidade $\left(\mathrm{E}_{\mathrm{C}}\right)$, sendo os maiores valores encontrados na espécie Corymbia maculata. Para madeiras de diferentes híbridos de Eucalyptus com menos de 10 anos de idade, SANTOS et al. [17] encontraram valores de resistência à compressão que variavam em uma faixa de $384,5 \mathrm{kgf} / \mathrm{cm}^{2}$ à $549,2 \mathrm{kgf} / \mathrm{cm}^{2}$, considerando $\mu_{\mathrm{EB}}$ de $0,638 \mathrm{~g} / \mathrm{cm}^{3}$ e $0,753 \mathrm{~g} / \mathrm{cm}^{3}$.

Tabela 2: Resumo estatístico e valores médios para os parâmetros dos ensaios de compressão paralela às fibras e dureza Janka das espécies florestais estudadas.

\begin{tabular}{ccc}
\hline & Compressão Paralela às Fibras & \\
\hline Espécie & Fc $\left(\mathbf{k g f} / \mathbf{c m}^{2}\right)$ & Ec $\left(\mathbf{k g f} / \mathbf{c m}^{2}\right)$ \\
\hline Corymbia maculata & $667,7(128,2) \mathrm{a}$ & $294.873,0(40.850,5) \mathrm{a}$ \\
\hline Eucalyptus dunnii & $450,2(193,2) \mathrm{a}$ & $262.507,0(15.633,2) \mathrm{a}$ \\
\hline Eucalyptus saligna & $627,2(71,2) \mathrm{a}$ & $228.127,0(55.186,7) \mathrm{a}$ \\
\hline CV $(\%)$ & 25,73 & 18,54 \\
\hline $\mathrm{F}$ & $2,34^{\mathbf{n s}}$ & $2,08^{\mathbf{n s}}$ \\
\hline Espécie & Dureza Janka & $\mathbf{R a d i a l ~}\left(\mathbf{k g f} / \mathbf{c m}^{2}\right)$ \\
\hline Corymbia maculata & Tangencial $\left(\mathbf{k g f} / \mathbf{c m}^{2}\right)$ & $593,8(65,50) \mathrm{a}$ \\
\hline Eucalyptus dunnii & $664,6(80,68) \mathrm{a}$ & $499,5(41,13) \mathrm{b}$ \\
\hline Eucalyptus saligna & $483,1(19,87) \mathrm{b}$ & $506,5(38,78) \mathrm{b}$ \\
\hline CV $(\%)$ & $615,2(30,72) \mathrm{a}$ & 12,27 \\
\hline $\mathrm{F}$ & 15,82 & $6,59 *$ \\
\hline
\end{tabular}


Em que: valores entre parênteses apresentam o desvio padrão $\left(\mathrm{kgf} / \mathrm{cm}^{2}\right)$. Médias nas colunas acompanhadas de mesma letra não diferem entre si, conforme teste LSD Fisher, em $5 \%$ de probabilidade de erro. $*$ = difere estatisticamente $(\mathrm{P}<$ $0,05) ;{ }^{\mathbf{n s}}=$ não difere estatisticamente $(\mathrm{P}>0,05)$;

Um fato que pode justificar a menor resistência à compressão dos autores supracitados em relação ao determinado no presente estudo pode estar relacionado a idade da árvore, uma vez que, a madeira dos híbridos de Eucalyptus eram constituídos basicamente de lenho juvenil, enquanto as espécies deste estudo são consideradas de lenho adulto.

Ainda de acordo com a Tabela 2, nota-se a existência de diferenças significativas na dureza Janka nos dois planos (tangencial e radial), e a espécie Corymbia maculata apresentou os maiores valores de dureza, independente do plano anatômico. Considerando essa propriedade, e a classificação proposta por CARVALHO [14], pode-se enquadrar as espécies Corymbia maculata e Eucalyptus saligna como madeiras de alta dureza (500 à $\left.700 \mathrm{kgf} / \mathrm{cm}^{2}\right)$, enquanto o Eucalyptus dunnii encontra-se inserido na classe de madeiras com dureza média (300 à $500 \mathrm{kgf} / \mathrm{cm}^{2}$ ).

Analisando madeiras de Eucalyptus grandis e Eucalyptus cloeziana com 17 anos de idade, GONÇALEZ et al. [18] obtiveram valores de dureza Janka perpendicular de $531 \mathrm{kgf}$ e $1.133 \mathrm{kgf}$, respectivamente. Já ARAÚJO et al. [19], estudando madeiras de Eucalyptus sp., determinaram uma dureza Janka de aproximadamente $639,4 \mathrm{kgf} / \mathrm{cm}^{2}$.

Quanto a diferença de dureza nos dois planos anatômicos possivelmente está associada à localização da penetração da esfera (no tangencial a aplicação da carga ocorre sobre o lenho tardio, enquanto no plano radial, pode ocorrer penetração da esfera sobre o lenho tardio ou entre dois consecutivos), a qual acaba influenciando na carga aplicada pelo equipamento para realizar a penetração da esfera.

Em síntese, os maiores valores de dureza Janka e parâmetros relacionados à compressão paralela às fibras $\left(F_{C}\right.$ e $\left.E_{C}\right)$ possivelmente estão associados à elevada massa específica dessa espécie, em comparação com as outras duas, indicando que esta propriedade física em específico pode ser considerada um indicativo satisfatório a fim de verificar a qualidade da madeira.

\subsection{Caracterização tecnológica das madeiras deterioradas por térmitas}

Observa-se a existência de diferenças significativas para a perda de massa (PM) das espécies estudadas (Tabela 3). A espécie com maior índice de deterioração pela ação de térmitas Nasutitermes sp. foi o Eucalyptus dunnii, sendo que o Eucalyptus saligna e a Corymbia maculata apresentaram baixa deterioração, não diferindo significativamente. Quanto a espécie utilizada como padrão de comparação, PAES et al. [20] e ALENCAR et al. [21] obtiveram perdas de massa de 4,54\% e 3,78\%, respectivamente, para a madeira de Pinus sp submetida ao ataque de Nasutitermes corniger, portanto, ambas inferiores ao encontrado.

Tabela 3: Resumo estatístico e valores percentuais médios de perda de massa das espécies deterioradas por térmitas $\mathrm{Na}$ sutitermes $\mathrm{sp}$.

\begin{tabular}{cc}
\hline Espécie & Perda de massa - PM (\%) \\
\hline Pinus elliottii $^{* *}$ & 7,31 \\
\hline Corymbia maculata $^{*}$ & $5,81(4,21) \mathrm{a}$ \\
\hline Eucalyptusdunnii & $17,21(5,41) \mathrm{b}$ \\
\hline Eucalyptus saligna & $3,68(0,64) \mathrm{a}$ \\
\hline $\mathrm{CV}(\%)$ & 79,51 \\
\hline $\mathrm{F}$ & $59,78^{*}$ \\
\hline
\end{tabular}

Em que: valores entre parênteses apresentam o desvio padrão (\%). Médias acompanhadas de mesma letra não diferem entre si, conforme teste LSD Fisher, em 5\% de probabilidade de erro. $*=$ difere estatisticamente $(\mathrm{P}<0,05) ;{ }^{\text {ns }}=$ não difere estatisticamente $(\mathrm{P}>0,05)$; ** : espécie de referência utilizada no ensaio, conforme recomendação da norma ASTM D 3345 (2008).

Em ensaio de laboratório com térmitas do mesmo gênero, STALLBAUN et al. [2] obtiveram uma perda de massa de aproximadamente $1,22 \%$ para a madeira de Sclerolobium paniculatum, enquanto PAES et al. [22], submetendo cinco espécies florestais ao ensaio de preferência alimentar com Nasutitermes corniger obtiveram perdas de massa médias de: 39,76\% (Acacia mangium); 17,82\% (Casuarina sp.); 22,26\% (Eucalyptus cloeziana); 13,55\% (Tectona grandis); 22,73\% (Corymbia torelliana), comprovando a variabilidade dos hábitos alimentares de térmitas em função de diferentes espécies. 
Além de parâmetros como massa específica e porosidade da madeira, OLIVEIRA et al. [3] argumentaram que a composição química tem papel fundamental na durabilidade natural da mesma, principalmente pela quantidade e qualidade dos extrativos ou compostos secundários da madeira. Isso permite inferir que as menores perdas de massa encontradas para as espécies de Eucalyptus saligna e Corymbia maculata possivelmente estão associadas à massa específica e composição dos extrativos.

A Tabela 4 mostra que não há diferença significativa entre as médias dos tratamentos do grupo controle e deterioradas por térmitas Nasutitermes, para os parâmetros de resistência à compressão $\left(\mathrm{F}_{\mathrm{C}}\right)$ e módulo de elasticidade $\left(\mathrm{E}_{\mathrm{C}}\right)$, para todas as folhosas. Observou-se redução no parâmetro $\mathrm{F}_{\mathrm{C}}$ para todas as espécies $(2,83 \%$ - Corymbia maculata; 5,82\% - Eucalyptus dunnii; 7,84\% - Eucalyptus saligna). Já para o $\mathrm{E}_{\mathrm{C}}$, obteve-se um aumento de 5,75\% para a Corymbia maculata, e decréscimo para o Eucalyptus dunnii $(1,76 \%)$ e Eucalyptus saligna $(1,54 \%)$.

Tabela 4: Resumo estatístico e valores médios dos parâmetros relacionados ao ensaio de compressão paralela às fibras para as madeiras deterioradas por térmitas.

\begin{tabular}{|c|c|c|c|}
\hline \multicolumn{4}{|c|}{ Resistência à Compressão - $\mathbf{F}_{\mathrm{C}}\left(\mathbf{k g f} / \mathrm{cm}^{2}\right)$} \\
\hline Grupo & Corymbia maculata & Eucalyptus dunnii & Eucalyptus saligna \\
\hline Controle & $667,7(128,2) \mathrm{a}$ & $450,2(193,2)$ a & $627,2(71,2) \mathrm{a}$ \\
\hline Deteriorada & $648,8(53,31) \mathrm{a}$ & $424,0(166,6) \mathrm{a}$ & $578,0(69,0) \mathrm{a}$ \\
\hline $\mathrm{CV}(\%)$ & 12,74 & 37,66 & 11,62 \\
\hline $\mathrm{F}$ & $0,07^{\mathrm{ns}}$ & $0,04^{\mathrm{ns}}$ & $0,99^{\mathrm{ns}}$ \\
\hline \multicolumn{4}{|c|}{ Módulo de Elasticidade - $\mathbf{E}_{\mathbf{C}}\left(\mathrm{kgf} / \mathrm{cm}^{2}\right)$} \\
\hline Grupo & Corymbia maculata & Eucalyptus dunnii & Eucalyptus saligna \\
\hline Controle & $294.873,0(40.850,5) \mathrm{a}$ & $262.507,0(15.633,2) \mathrm{a}$ & $228.127,0(55.186,7) \mathrm{a}$ \\
\hline Deteriorada & $311.828,0(55.054,1) \mathrm{a}$ & $257.879,0(40.872,0) \mathrm{a}$ & $224.606,0(15.281,9) \mathrm{a}$ \\
\hline $\mathrm{CV}(\%)$ & 15,24 & 12,36 & 16,58 \\
\hline $\mathrm{F}$ & $0,20^{\mathrm{ns}}$ & $0,03^{\mathrm{ns}}$ & $0,02^{\mathrm{ns}}$ \\
\hline
\end{tabular}

Em que: valores entre parênteses apresentam o desvio padrão $\left(\mathrm{kgf} / \mathrm{cm}^{2}\right)$. Médias nas colunas acompanhadas de mesma letra não diferem entre si, conforme teste LSD Fisher, em 5\% de probabilidade de erro. ${ }^{*}=$ difere estatisticamente $(\mathrm{P}<$ $0,05) ;{ }^{\mathbf{n s}}=$ não difere estatisticamente $(\mathrm{P}>0,05)$;

Na Tabela 5 nota-se a ausência de modificações significativas na dureza Janka, no plano tangencial, das madeiras das três espécies florestais. Enquanto na espécie Corymbia maculata ocorreu um aumento (3,55\%), o Eucalyptus dunnii e o Eucalyptus saligna apresentaram redução na dureza $(2,40 \%$ e $2,61 \%$, respectivamente). Já no plano radial, verifica-se um aumento significativo somente na dureza dos corpos de prova deteriorados em comparação ao grupo controle $(22,94 \%)$ para a espécie Eucalyptus saligna, enquanto para as outras espécies observou-se comportamentos distintos para os tratamentos (aumento de 5,88\% para a espécie Corymbia maculata e redução de 13,77\% para o Eucalyptus dunnii).

Tabela 5: Resumo estatístico e valores médios de dureza Janka para as madeiras deterioradas por térmitas.

\begin{tabular}{|c|c|c|c|}
\hline \multicolumn{4}{|c|}{ Plano Longitudinal Tangencial $\left(\mathrm{kgf} / \mathrm{cm}^{2}\right)$} \\
\hline Grupo & Corymbia maculata & Eucalyptus dunnii & Eucalyptus saligna \\
\hline Controle & $664,6(80,7)$ a & $483,1(19,9) \mathrm{a}$ & $615,2(30,7) \mathrm{a}$ \\
\hline Deteriorada & $688,2(78,6) \mathrm{a}$ & $471,5(46,2) \mathrm{a}$ & $593,0(43,8) \mathrm{a}$ \\
\hline $\mathrm{CV}(\%)$ & 11,46 & 7,14 & 6,02 \\
\hline $\mathrm{F}$ & $0,31^{\mathrm{ns}}$ & $0,27^{\mathrm{ns}}$ & $0,80^{\mathrm{ns}}$ \\
\hline \multicolumn{4}{|c|}{ Plano Longitudinal Radial $\left(\mathrm{kgf} / \mathrm{cm}^{2}\right)$} \\
\hline Grupo & Corymbia maculata & Eucalyptus dunnii & Eucalyptus saligna \\
\hline Controle & $593,8(62,5)$ a & $499,5(41,1) \mathrm{a}$ & $506,5(38,8) \mathrm{b}$ \\
\hline Deteriorada & $628,7(76,9) \mathrm{a}$ & $430,7(78,3) \mathrm{a}$ & $622,7(41,3) \mathrm{a}$ \\
\hline $\mathrm{CV}(\%)$ & 11,4 & 14,88 & 12,74 \\
\hline $\mathrm{F}$ & $0,87^{\mathrm{ns}}$ & $3,02^{\mathrm{ns}}$ & $21,00^{*}$ \\
\hline
\end{tabular}


Em que: valores entre parênteses apresentam o desvio padrão $\left(\mathrm{kgf} / \mathrm{cm}^{2}\right)$. Médias nas colunas acompanhadas de mesma letra não diferem entre si, conforme teste LSD Fisher, em 5\% de probabilidade de erro. * $=$ difere estatisticamente $(\mathrm{P}<$ $0,05) ;{ }^{\text {ns }}=$ não difere estatisticamente $(\mathrm{P}>0,05)$;

Essas variações na dureza Janka (planos radial e tangencial) e nos parâmetros relacionados aos módulos de elasticidade e resistência à compressão (compressão paralela às fibras), considerando as madeiras do grupo controle e as que foram deterioradas por térmitas Nasutitermes, podem estar associadas com a perda de massa, e consequente degradação dos constituintes primários (celulose, hemicelulose e lignina), quebra de ligações entre os átomos e surgimento de galerias no interior e superfície das madeiras atacadas.

Conforme CORASSA et al. [23], a construção de galerias no interior da madeira, devido ao ataque de térmitas, tende a diminuir a resistência mecânica desse material. BRAZ et al. [24] relataram que a resistência mecânica da madeira está diretamente correlacionada aos seus teores de lignina e celulose. Logo, se os constituintes químicos são removidos simultaneamente, a madeira tende a sofrer redução na sua resistência mecânica.

Contudo, RODRIGUES et al. [25] argumentaram que devido sua complexidade e heterogeneidade, a madeira é passível de sofrer diversas variações em sua estrutura. Portanto, variações nas propriedades tecnológicas (com ênfase nas mecânicas) possivelmente estão associadas a questões anatômicas, as quais podem apresentar variação entre diferentes espécies e indivíduos de uma mesma espécie [26], conforme observado nos ensaios de dureza Janka (plano tangencial e radial) e compressão paralelas às fibras $\left(\mathrm{F}_{\mathrm{C}}\right.$ e $\left.\mathrm{E}_{\mathrm{C}}\right)$.

Assim, estudos que abordem a preferência alimentar de determinadas espécies de térmitas, bem como, análises que a deterioração desses xilófagos causam nas características tecnológicas, anatômicas e na composição química, tornam-se de suma importância para a compreensão do comportamento da madeira. Isso possibilita a seleção de uma espécie florestal adequada e correta utilização desse material, considerando o uso ao qual se destina.

\section{CONCLUSÕES}

A espécie Corymbia maculata possui parâmetros tecnológicos que lhe conferem maior resistência mecânica quando comparada com Eucalyptus dunnii e o Eucalyptus saligna, estando possivelmente relacionada com a sua maior massa específica básica $\left(\mu_{\mathrm{EB}}\right)$. A espécie que apresentou mais susceptibilidade à deterioração por térmitas Nasutitermes sp., devido à preferência alimentar dos mesmos, foi o Eucalyptus dunnii, podendo tal fato estar atrelado a sua menor massa específica básica $\left(\mu_{\mathrm{EB}}\right)$, em conjunto com a sua composição química, a qual possui influência direta na resistência à deterioração biológica da madeira.

A deterioração causada por térmitas causou alterações nas variáveis relacionadas ao ensaio de compressão paralela às fibras (resistência à compressão $-\mathrm{F}_{\mathrm{C}}$ e módulo de elasticidade $-\mathrm{E}_{\mathrm{C}}$ ), e na dureza Janka, nos planos anatômicos radial e tangencial.

\section{AGRADECIMENTOS}

Os autores agradecem a CMPC Celulose Rio-grandense pela doação das madeiras de Corymbia maculata, Eucalyptus dunnii e Eucalyptus saligna utilizadas na realização deste estudo, e à Coordenação de Aperfeiçoamento de Pessoal de Nível Superior (CAPES) pela concessão da Bolsa de Doutorado.

\section{BIBLIOGRAFIA}

[1] MOTTA, J. P., OLIVEIRA, J. T. S., PAES, J. B., et al., "Resistência natural da madeira de Tectona grandis em ensaio de laboratório", Ciência Rural, Santa Maria, v.43, n.8, pp.1393-1398, 2013.

[2] STALLBAUN, P. H., BARAUNA, E. E. P., PAES, J. B., et al., "Resistência natural da madeira de Sclerolobium paniculatum Vogel a cupins em condições de laboratório", Floresta e Ambiente, Seropédica, v. 24, 2017.

[3] OLIVEIRA, J. T. S., SOUZA, L. C., DELLA LUCIA, R. M., et al., "Influência dos extrativos na resistência ao apodrecimento de seis espécies de madeira", Revista Árvore, Viçosa-MG, v. 29, n. 5, pp. 819-826, 2005 .

[4] HORNBURG, K. F., ELEOTÉRIO, J. R., BAGATTOLLI, T. R., et al., "Qualidade das toras e da madeira serrada de seis espécies de eucalipto cultivadas no litoral de Santa Catarina", Scientia Forestalis, Piracicaba, v. 40, n. 96, pp. 463-471, 2012. 
[5] SOUZA, C. C., MOREIRA, A. A., SCHIMITH, R. S., et al., "Técnicas de sensoriamento remoto como subsídios aos estudos de florestas implantadas no Brasil - uma revisão bibliográfica", Ciência Florestal, Santa Maria, v. 17, n. 4, pp. 409-417, 2007.

[6] DIAS JÚNIOR, A. F., SANTOS, P. V., PACE, J. H. C., et al., "Caracterização da madeira de quatro espécies florestais para uso em movelaria", Ciência da Madeira, Pelotas, v. 4, n. 1, pp. 93-107, 2013.

[7] ARAÚJO, B. H. P., SOUZA, M. A. R., NASCIMENTO, H. E. M., et al., "Propriedades físicas da madeira de Calycophyllum spruceanum Benth. em função do diâmetro e da posição (base e topo) no fuste", Scientia Forestalis, Piracicaba, v. 44, n. 111, pp. 759-768, 2016.

[8] LOGSDON, N. B., FINGER, Z., ROSA, L. M. "Caracterização da madeira de Vochysia guianensis Aubl”, Engenharia Civil, n.29, pp. 57-69, 2007.

[9] AMERICAN SOCIETY FOR TESTING AND MATERIALS (ASTM). "Standard test method for laboratory evaluation of wood and other cellulosic materials for resistance to térmites". ASTM D 3345-74. West Conshohocke, 2008.

[10] AMERICAN SOCIETY FOR TESTING AND MATERIALS (ASTM). "Standard test methods for small clear specimens of timber". ASTM D 143 - 94. Philadelphia, 2014.

[11] STEEL, R. G. D., TORRIE, J. H. "Principles and procedures of statistic: a biometrical approach", 2. ed. New York, McGrawHill, 1980. 633 p.

[12] MARTINS, M., SILVA, J. R. M., LIMA, J. T., et al., "Simulação em uso dos pisos de madeira simulação em uso dos pisos de madeira de Eucalyptus sp e Corymbia maculata", Cerne, Lavras, v. 19, n. 1, pp. 151-156, 2013.

[13] BATISTA, D. C., KLITZKE, R. J., SANTOS, C. V. T. "Massa específica básica e retratibilidade da madeira de clones de três espécies de Eucalyptus", Ciência Florestal, Santa Maria, v. 20, n. 4, p. 665-674, 2010.

[14] CARVALHO, A. "Madeiras portuguesas, estrutura anatômica, propriedades utilizações". Instituto Florestal, v. 1, 1996.

[15] TREVISAN, R., HASELEIN, C.R., MELO, R.R., et al., "Variação radial da massa específica básica da madeira de Eucalyptus grandis W. Hill ex Maiden”, Floresta, Curitiba, n.3, pp.553-559, 2008.

[16] MATTOS, B. D., GATTO, D. A., STANGERLIN, D. M., et al., "Variação áxil da densidade básica da madeira de três espécies gimnospermas", Revista Brasileira de Ciências Agrárias, Recife, v. 6, n. 1, p. 121126, 2011.

[17] SANTOS, J. A., SANTOS, J., BORRALHO, N., et al., "Caracterização das potencialidades de utilização da madeira de híbridos de eucalipto". Silva Lusitana, Lisboa, v. 16, n. 1, p. 63-81, 2008.

[18] GONÇALEZ, J. C., BREDA, L. C. S., BARROS, J. F. M., et al., "Características tecnológicas das madeiras de Eucalyptus grandis W. Hill ex Maiden e Eucalyptus cloeziana F. Muell visando ao seu aproveitamento na indústria moveleira", Ciência Florestal, Santa Maria, v. 16, n. 3, p. 329-341, 2006.

[19] ARAÚJO, S. O., VITAL, B. R., MENDOZA, Z. M. S. H., et al., "Propriedades de madeiras termorretifcadas de Eucalyptus grandis e SP", Scientia Forestalis, Piracicaba, v. 40, n. 95, p. 327-336, 2012.

[20] PAES, J. B., SOUZA, A. D., LIMA, C. R., et al., "Eficiência dos óleos de Nim e Mamona contra cupins xilófagos em ensaio de alimentação forçada", Cerne, Lavras, v. 16, n. 1, pp. 105-113, 2010.

[21] ALENCAR, F. H. H., PAES, J. B., BAKKE, O. A., et al., "Resistência natural da madeira de sabiá (Mimosa caesalpiniifolia Benth.) a cupins subterrâneos”, Revista Caatinga, Mossoró, v. 24, n. 1, pp. 57-64, 2011.

[22] PAES, J. B., GUERRA, S. C. S., SILVA, L. F., et al., "Efeito do teor de extrativos na resistência natural de cinco madeiras ao ataque de cupins xilófagos", Ciência Florestal, Santa Maria, v. 26, n. 4, p. 1259-1269, 2016.

[23] CORASSA, J. N., PIRES, E. M., ANDRADE NETO, V. R., et al., "Témitas associados à degradação de cinco espécies florestais em campo de apodrecimento", Floresta e Ambiente, Seropédica, v. 21, n. 1, pp. 7884, 2014. 
[24] BRAZ, R. L., OLIVEIRA, J. T. S., ROSADO, A. M., et al., "Caracterização anatômica, física e química da madeira de clones de Eucalyptus cultivados em áreas sujeitas à ação de ventos", Ciência da Madeira, Pelotas, v. 5, n. 2, pp. 127-137, 2014.

[25] RODRIGUES, D. A., SILVEIRA, A. P., CASTELLO, P. A. R. "Determinação de propriedades físicomecânicas da madeira de cinco espécies madeireiras da Amazônia Meridional”, Scientific Electronic Archives, v. 7, pp. 52-58, 2014.

[26] EVANGELISTA, W. V., SILVA, J. C., DELlA LUCIA, R. M., et al., "Propriedades físico-mecânicas da madeira de Eucalyptusurophylla S.T. Blake no sentido radial e longitudinal”, Ciência da Madeira, Pelotas, v. 1, n. 2, pp. 1-19, 2010. 